\title{
Studies on $\eta$ meson production in dp collisions at the magnetic spectrometer ANKE
}

\author{
Christopher Fritzsch ${ }^{* \dagger}$ \\ WWU Münster / ANKE Collaboration \\ E-mail: c.fritzscheuni-muenster.de
}

\author{
Alfons Khoukaz \\ WWU Münster / ANKE Collaboration \\ E-mail: khoukaz@uni-muenster.de \\ Daniel Schröer \\ WWU Münster / ANKE Collaboration \\ E-mail: d_schr13@uni-muenster.de
}

\begin{abstract}
The magnetic spectrometer ANKE is an internal fixed target experiment at the accelerator and storage ring COSY, located at the Forschungszentrum Jülich in Germany, to study the properties and interaction of hadrons in a medium energy regime. For this purpose, COSY provides (un)polarized deuteron and proton beams with momenta between $0.3 \mathrm{GeV} / c$ and $3.7 \mathrm{GeV} / c$. Furthermore, the design of the ANKE detector setup with its three dipole magnets is very well suited for the detection and reconstruction of charged particles generated even at small scattering angles in reactions close to threshold. Current investigations focus on the behaviour of the $\eta$ meson production process as well as on the study of angular distributions in the reactions $d+p \rightarrow{ }^{3} \mathrm{He}+\eta$ and $p+d \rightarrow d+\eta+p_{\text {spectator }}$. The recent analysis status for both reactions will be presented and discussed.
\end{abstract}

The 26th International Nuclear Physics Conference

11-16 September, 2016

Adelaide, Australia

\footnotetext{
* Speaker.

$\dagger$ This work has been supported by the COSY-FFE program of the Forschungszentrum Jülich and the Deutsche Forschungsgemeinschaft (DFG) through the Research Training Group "GRK 2149: Strong and Weak Interactions from Hadrons to Dark Matter".
} 


\section{Introduction}

The total and differential cross section of the reaction $d+p \rightarrow \mathrm{He}+\eta$ are of special interest since they differ strongly from a pure phase space behaviour close to threshold. Assuming there is no final state interaction between the ${ }^{3} \mathrm{He}$ nucleus and the $\eta$ meson one would expect that in good approximation the total cross section will increase proportional to $\sqrt{Q}$ - the square root of the excess energy Q. In contrast, the total cross section shows a steep enhancement in the near threshold region within the first $\mathrm{MeV}$ ( $\mathrm{cf}$ Figure 1), which can be explained by a nearby pole in the scattering amplitude $[1,2]$.

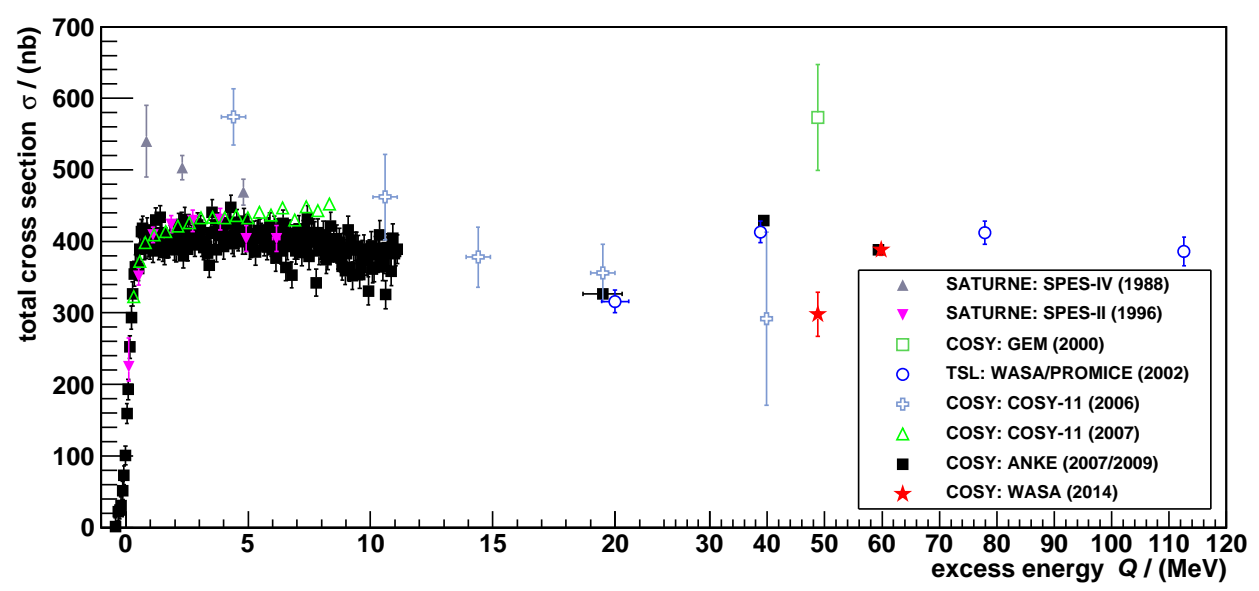

Figure 1: Total cross section $\sigma$ of the reaction $d+p \rightarrow \mathrm{He}+\eta$ [3]-[12]. Note that the systematic uncertainties are not shown and that the scale in the excess energy $Q$ is broken at $Q=25 \mathrm{MeV}$.

In addition to that, studies on the asymmetry (cf. Figure 2 and Equation (3.1)) of the differential cross section as a function of the final state momentum $p_{\eta}$ of the $\eta$ meson show a distinct effect of an s- and p-wave interference, which leads to a rapid variation of the relative phase. This effect in combination with the non-pure phase space behaviour of the total cross section close to threshold are indications for an unexpected strong final state interaction (FSI) between the ${ }^{3} \mathrm{He}$ nucleus and the $\eta$ meson which could be associated with a possible quasi bound state of the ${ }^{3} \mathrm{He} \eta$-system $[1,2]$.

To further investigate this, current studies on high precision data from the magnetic spectrometer ANKE focus on the extraction of total and differential cross sections up to an excess energy of $Q=15 \mathrm{MeV}$. On the one hand the total cross sections in combination with current studies by the WASA-at-COSY experiment will clarify the behaviour of the total cross sections over a huge excess energy range (up to $80 \mathrm{MeV}$ ) and on the other hand the differential cross

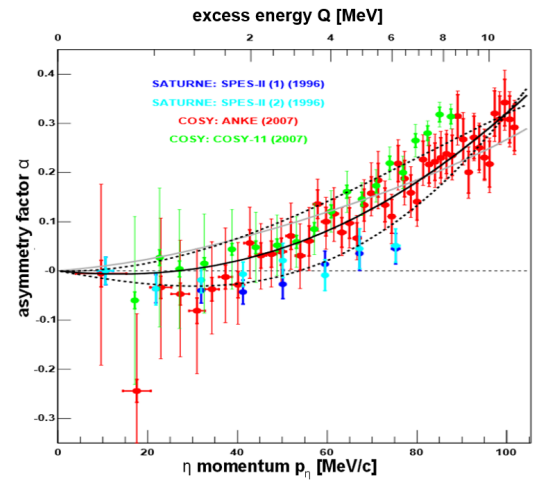

Figure 2: Asymmetry parameter $\alpha$ of the differential cross sections of the reaction $d+p \rightarrow \mathrm{He}+\eta$ up to an excess energy of $Q=11 \mathrm{MeV}$. sections will significantly improve the accuracy of the data base of the asymmetry parameter $\alpha$ to get a better understanding of the strength of the s- and p- 
wave interference and contribution from possible higher partial waves. Assuming the FSI ansatz to be correct, the non-pure phase space behaviour of the total cross section close to threshold should also be seen in other production channels. This has been tested by different facilities. Nevertheless, none of these measurements could clearly prove whether the $\eta$-nucleus system is a bound or virtual state [13]. In order to further investigate the properties of a possible formation of $\eta$-mesic nuclei, a beam time was realized at ANKE to study the $p+d \rightarrow p_{\text {spec }}+d+\eta$ reaction. The behaviour of the total cross section will allow to determine the scattering length $a_{d \eta}$ by using a s-wave final state ansatz whereas the differential cross section will clarify the validity of this s-wave assumption.

\section{Experimental Setup}

The magnetic spectrometer ANKE ("Apparatus for Studies of Nucleon and Kaon Ejectiles") is an internal fixed target experiment at the accelerator and storage ring COSY ("COoler SYnchrotron"), located at the Forschungszentrum Jülich in Germany. COSY provides polarized as well as unpolarized hydrogen or deuterium beams, which can be accelerated to final momenta up to $3.7 \mathrm{GeV} / c$. The two available cooling systems (stochastic and electron cooling) allow a momentum resolution of $\Delta p / p \approx 10^{-4}$.

The schematic picture of the magnetic spectrometer ANKE is shown in Figure 3. It consists of three dipole magnets, an internal target and three detection systems. The COSY beam direction is from the left hand side and will be deflected by the first dipole magnet D1 into the scattering chamber. There it can interact with the internal cluster jet target (unpolarized hydrogen or deuterium). The produced ejectiles will be separated by the second dipole magnet D2 due to their electrical charge into the negative or positive detection system (Nd- and $\mathrm{Pd}$-system).

In addition to that, there is a third detection system, the so called forward detection system (Fd-system), which was designed to detect very fast or heavy positively charged particles like protons, deuterons or ${ }^{3} \mathrm{He}$ nuclei. These particles will be deflected by D2 just into small laboratory solid angle elements near the beam pipe. Particles which do not interact with the internal target will be deflected by D2 and D3 onto the nominal ring orbit. A special feature of this experiment is the movable D2 magnet, which can be shifted in $\mathrm{x}$-direction so one is able to obtimize the geometrical acceptance for each reaction one would like to investigate. Both the ${ }^{3} \mathrm{He}+\eta$ and

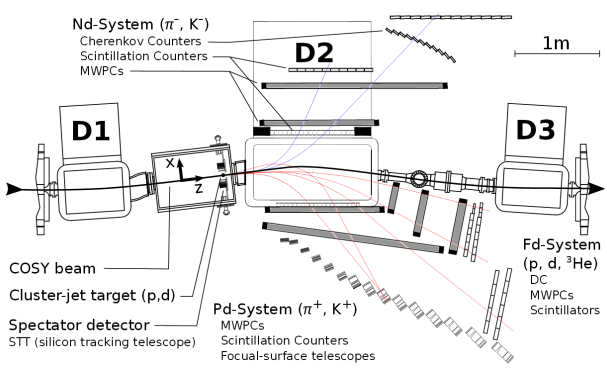

Figure 3: Schematic picture of the magnetic spectrometer ANKE with its main components (three dipole magnets, internal target, and three detection systems).

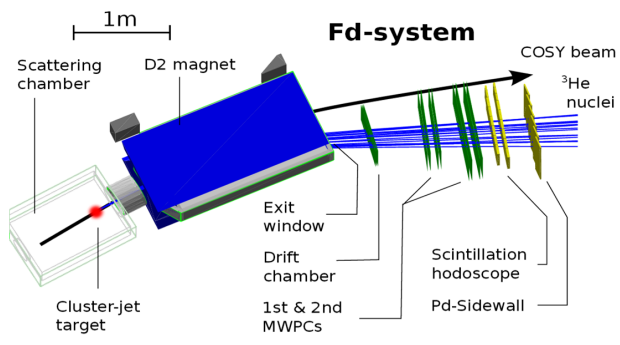

Figure 4: Schematic picture of the Fd-system with its main components (three multiwire chambers (green) and three layers of scintillator hodoscopes (yellow). $p_{\text {spec }}+d+\eta$ production channel use the Fd-system for particle identification. It consists of one multiwire drift and two multiwire proportional cham- 
bers for track reconstruction and three layers of scintillator hodoscopes, consisting of eight, nine and six scintillator stripes, for energy loss and time-of-flight measurements.

\section{The $d+p \rightarrow{ }^{3} \mathrm{He}+\eta$ production channel}

The data used for this analysis have been taken at 18 different beam momenta between $p_{d}=$ $3120.17 \mathrm{MeV} / c$ and $p_{d}=3204.16 \mathrm{MeV} / c$ using the supercycle mode, which means that the beam momentum alternates between up to seven different beam momenta within one supercycle.

Due to two body kinematics, it is not necessary to detect both final state particles. Accordingly, the identification of the $d+p \rightarrow{ }^{3} \mathrm{He}+\eta$ reaction is achieved by detecting the ${ }^{3} \mathrm{He}$ nuclei in the $\mathrm{Fd}$ system using the reconstructed momentum and the energy loss information in the scintillator hodoscopes. As shown in Figure 5 a clear characteristic ${ }^{3} \mathrm{He}$ energy loss band is visible. By applying software cuts on this energy loss band most of the competing background reactions vanish. A missing mass analysis shows then a distinct signal at the mass of the $\eta$-meson for each of the 15 beam momenta

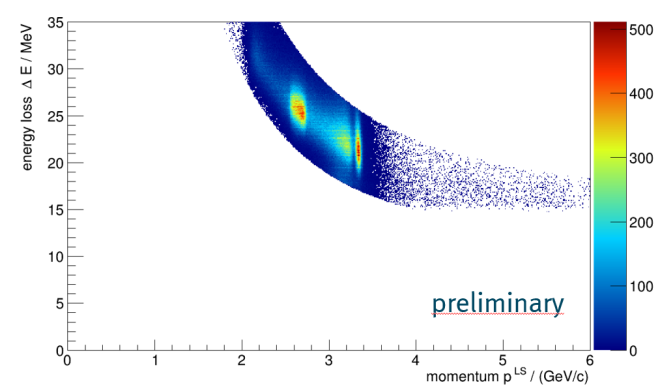

Figure 5: Preliminary energy loss information of the characteristic ${ }^{3} \mathrm{He}$-band of the plastic scintillator hodoscopes in the Fd-system. above the production threshold (cf. Figure 6). To get rid of the remaining background, a special analysis method is used. During the beam time also data at $p_{d}=3120.17 \mathrm{MeV} / c$, i.e. below the $\eta$ production threshold, were taken, which allow a model independent approach for the background description. Therefore, the sub-threshold data will be analyzed with the the above threshold labo-
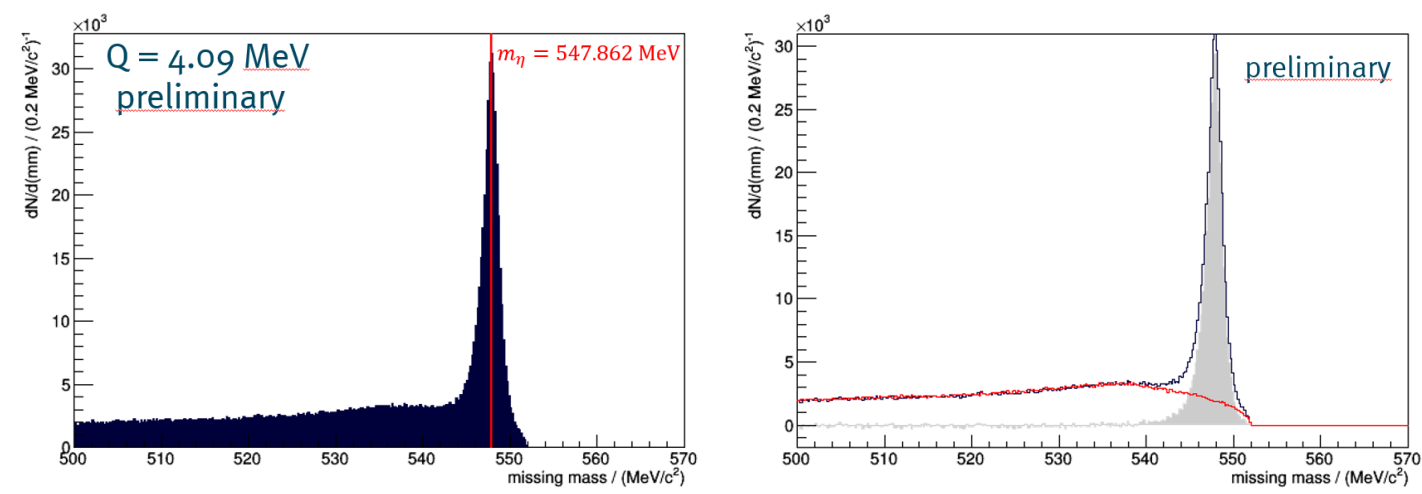

Figure 6: Left: Preliminary missing mass spectrum (filled blue) at $p_{d}=3158.71 \mathrm{MeV} / c$. The red vertical line represent the mass of the $\eta$-meson. Right: Corresponding preliminary subtracted missing mass spectrum (filled gray peak) using the sub-threshold data (red line).

ratory momentum, so that every event will be transformed to the "false" laboratory system, which leads to a shift of the kinematical limit in the missing mass spectrum. After subtraction a clear $\eta$ peak becomes visible with more than $10^{5}{ }^{3} \mathrm{He} \eta$ - events for each of the 15 above threshold beam momenta. The normalization was done using the dp-elastic scattering with a statistical uncertainty of $\Delta_{\text {stat }} \approx 1 \%$ and a systematic uncertainty of $\Delta_{\text {sys }} \approx 6 \%$ [14], which is an improvement by at 
least a factor of two compared to previous measurements. These luminosities were already used to determine double differential cross section for the double pion production in the channel $d+p \rightarrow{ }^{3} \mathrm{He}+\pi^{+}+\pi^{-}[15]$. This high precision normalization in combination with the high statistic of the $\eta$ production leads to a precise extraction of the total cross section of the ${ }^{3} \mathrm{He} \eta$ production channel (cf. Figure 7). The preliminary results show, as expected, a pretty flat behaviour over an excess energy range of $\Delta Q=15 \mathrm{MeV}$ and are also in good agreement with the results of previous measurements. Note here, that just the statistical uncertainties are shown.

Due to the high statistics and the full angular coverage of ANKE, a precise study of the angular distribution is also possible (cf. Figure 8). The anisotropy of the differential cross

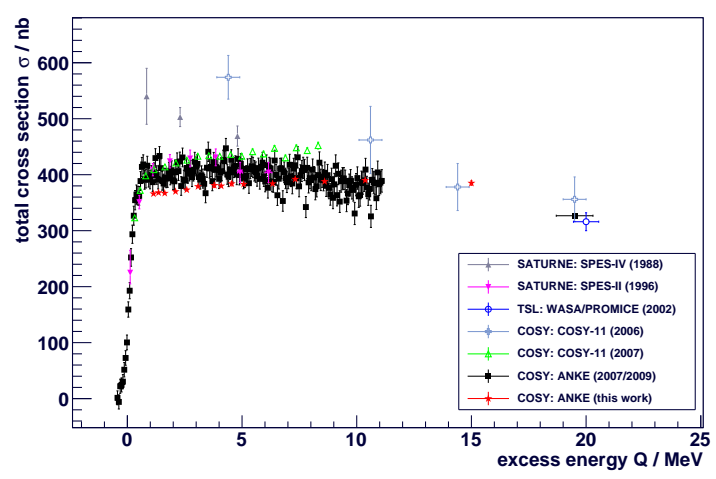

Figure 7: Preliminary results (red stars) of the total cross section $\sigma$ of the reaction $d+p \rightarrow{ }^{3} \mathrm{He}+\eta$. Note that the systematic uncertainties are not shown. sections $d \sigma / d \Omega$ regarding the forward and backward direction in the center of mass system can be expressed in terms of the asymmetry parameter $\alpha$, given by:

$$
\alpha=\left.\frac{d}{d \cos \left(\vartheta_{\eta}^{C M S}\right)} \ln \left(\frac{d \sigma}{d \Omega}\right)\right|_{\cos \left(\vartheta_{\eta}^{C M S}\right)=0} .
$$

The preliminary results of the asymmetry parameter $\alpha$ are shown in Figure 8 as blue stars. Note here, that due to the very preliminary status no uncertainties are shown. Nevertheless, a clear increase of the $\alpha$ value with increasing final state momentum (excess energy) is visible, which can be explained by introducing higher partial waves like a p-wave, starting at approximately $p_{f}^{C M S}=50 \mathrm{MeV} / c$. Furthermore, the absolute value of $\alpha$ suggest to be consistently positive at lower final state momenta $p_{f}^{C M S} \leq 40 \mathrm{MeV} / c$.
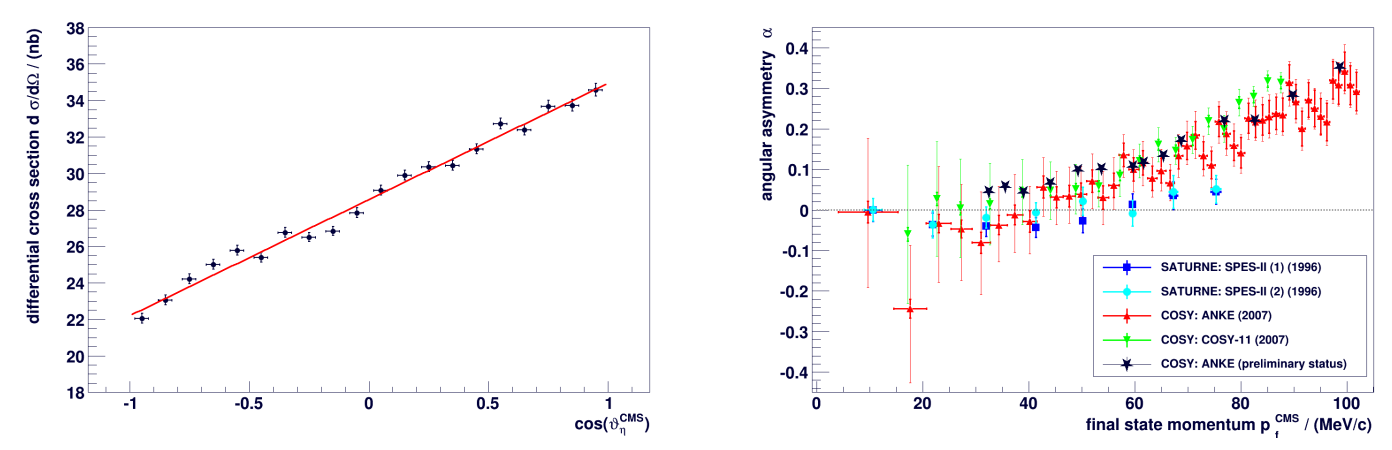

Figure 8: Left: Preliminary differential cross section of the for the ${ }^{3} \mathrm{He} \eta$ production channel at $p_{d}=$ $3168.05 \mathrm{MeV} / c$. The uncertainties in $d \sigma / d \Omega$ represent the statistical uncertainties. The red solid line is a linear fit to the angular distribution. Right: Preliminary results of the asymmetry factor $\alpha$ (blue stars) up to an excess energy of $Q=11 \mathrm{MeV}$. Due to the very preliminary status the uncertainties of the data points are not shown. 


\section{The $p+d \rightarrow d+\eta+p_{\text {spectator }}$ production channel}

For the measurement of the production channel $p+d \rightarrow d+\eta+p_{\text {spectator }}$ COSY provided proton beams with momenta of $p_{1}^{\text {beam }}=2.09 \mathrm{GeV} / c$ and $p_{2}^{\text {beam }}=2.25 \mathrm{GeV} / c$. Due to the fact, that the neutron within the target deuteron serves as an effective neutron target with the proton being a spectator particle, this production channel can be described as the quasi free reaction $p+n \rightarrow d+\eta$. This combination allows to extract total and differential cross section from threshold up to an excess energy of $Q=90 \mathrm{MeV}$. The reaction can be fully described using the missing mass method by detecting the spectator proton and the deuteron. The deuterons are detected in the Fd-system and selected via the energy loss information in the plastic scintillators of the hodoscopes. Due to the low
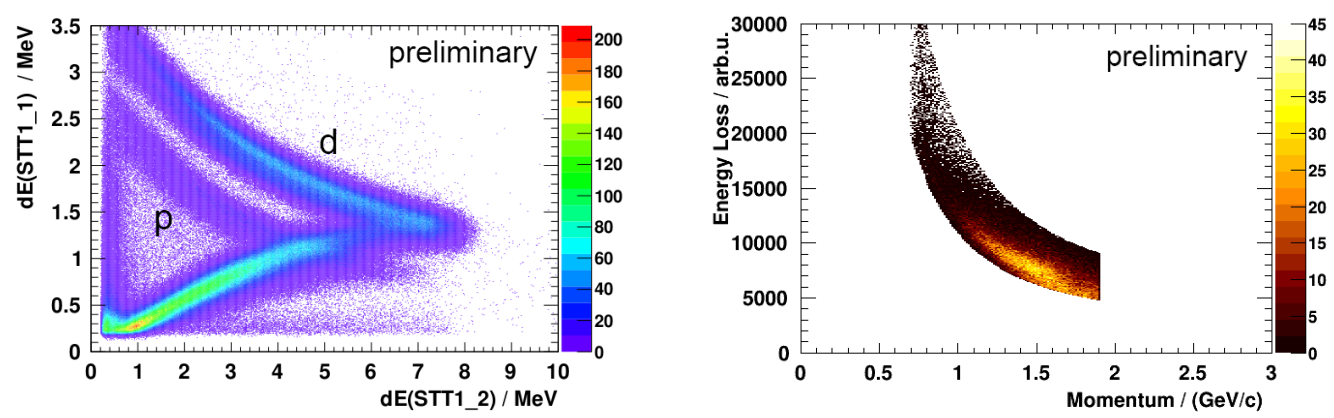

Figure 9: Left: Preliminary energy loss information by plotting the energy in the first against the energy loss in the second layer of one STT. Right: Preliminary energy loss information of the characteristic deuteron band of the plastic scintillator hodoscopes in the Fd-system.

momentum of the spectator protons (caused by their Fermi motion within the deuteron target), they would not reach the D2 magnet of the ANKE spectrometer. Therefore, the energy loss information of two Silicon Tracking Telescopes ("STT"), which were placed next to the interaction point, are used for the identification of the spectator protons. As shown in Figure 9 a clear deuteron energy
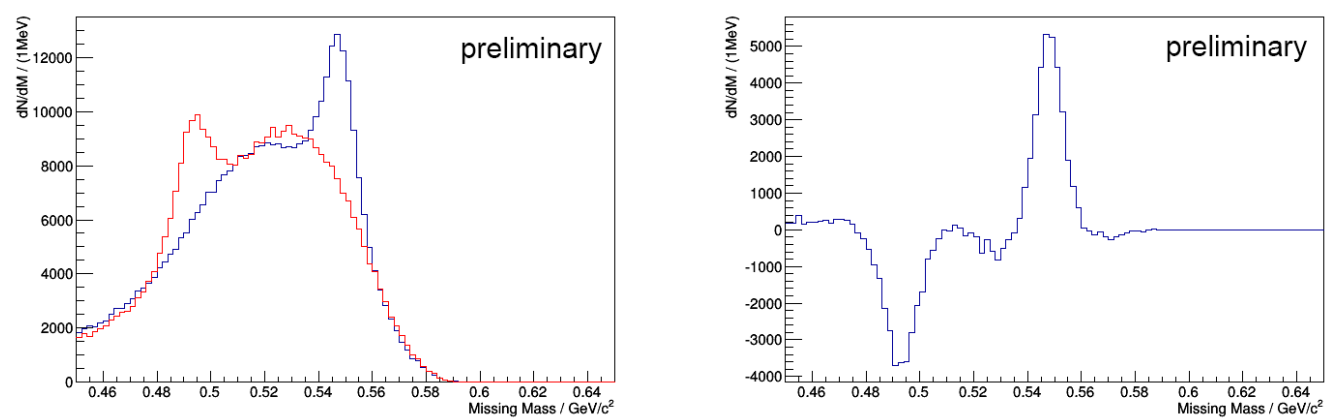

Figure 10: Left: Preliminary missing mass spectrum for both beam momenta ( $p_{1}^{\text {beam }}=2.09 \mathrm{GeV} / c$ data in blue and shifted $p_{2}^{\text {beam }}=2.25 \mathrm{GeV} / c$ in red). Right: Preliminary subtracted missing mass spectrum with two clear $\eta$ signals (positive and negative peak).

loss band, mainly from elastic scattering, and a proton band becomes visible. The identification 
of the deuterons is more challenging, due to a huge proton background. The energy loss of the deuterons and protons are quite similar in this momentum range, so that the determination of cut values is demanding. Thereby, the time-of-flight differences between positively charged pions from competing reactions in the Pd-system and particles in the Fd-system (e.g. $p+d \rightarrow d+\pi^{+}+X$ ) were used to determine accurate energy loss cut values.

Hence the proton background can be suppressed, so that the deuteron band becomes visible (cf. Figure 9). The remaining background is mainly caused by multi pion production. The chosen two different beam momenta allow also a model independent approach for the background subtraction. Therefore, the data of the higher beam momentum $p_{2}^{\text {beam }}$ will be analyzed with the "false" lower momentum $p_{1}^{\text {beam }}$, which leads to a shift of both the $\eta$-peak and the kinematical limit in the missing mass spectrum (cf. Figure 10). After subtraction two distinct $\eta$ signals with approximately $10^{5} \eta$ events are visible.

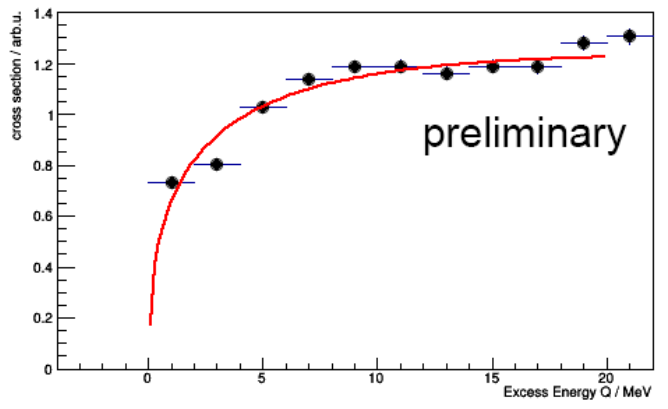

Figure 11: Preliminary results of the total cross section $\sigma$ of the reaction $p+d \rightarrow p_{\text {spectator }}+d+\eta$. Note that the systematic uncertainties are not shown. The red line represent a s-wave final state ansatz fit to the data.

First preliminary results of the total cross section are shown in Figure 11. The red line represent a fit to the data using the s-wave final state ansatz:

$$
\frac{p_{i}}{p_{f}} \cdot \frac{d \sigma}{d \Omega}=|f|^{2}=\left|f_{s} \cdot \mathrm{FSI}\right|^{2}
$$

with

$$
\mathrm{FSI}=\frac{1}{1-i \cdot a \cdot p_{f}}
$$

and $p_{i}\left(p_{f}\right)$ being the initial (final) state momentum, $f_{s}$ the to good approximation constant production amplitude, and $a$ the scattering length. Thereby, the fit to the unnormalized total cross section using (4.1) and (4.2) describes the data nicely. In the final analysis e.g. the finite beam momentum distribution has to be considered.

\section{Summary and Outlook}

Due to the high statistic of the $d+p \rightarrow{ }^{3} \mathrm{He}+\eta$ reaction in combination with the precise normalization using the $d p$-elastic scattering, precise total cross section up to an excess energy of $Q=15 \mathrm{MeV}$ will be extracted. These preliminary results are also consistent with previous measurements. Furthermore, the full coverage of the ANKE spectrometer in $\cos \left(\vartheta_{\eta}^{C M S}\right)$ ensures an accurate investigation of the angular distribution. The shown anisotropy of the differential cross sections regarding to the forward and backward direction can be described by an asymmetry parameter $\alpha$. The results of $\alpha$ legitimate the introduction of a p-wave starting at approximately $p_{f}^{C M S}=50 \mathrm{MeV} / c$. Given that the cross section is proportional to the absolute square of the scattering amplitude, the information of the relative phase variation of the s-wave is lost. Nevertheless, the interference of 
the s-wave and the starting p-wave at higher final state momenta will regain this information. Due to the fact that some analysis steps, like a careful determination of the uncertainties for example, are still in progress, the shown results are in a preliminary status.

In case of the $p+d \rightarrow d+\eta+p_{\text {spectator }}$ production channel it is possible to detect and reconstruct the deuterons in the Fd-system and the spectator protons in the STT's of the ANKE spectrometer, which leads to an overall $\eta$ quantity of approximately $10^{5}$. Also a scattering length will be extracted by fitting the s-wave final state ansatz to the final total cross section of the reaction. A precise luminosity determination using the elastic scattering and the determination of the differential cross sections is in progress, which will be used to clarify the range of validity of the s-wave final state ansatz.

\section{References}

[1] C. Wilkin, Near-Threshold Production of $\eta$-Mesons, Phys. Rev. C 47, R938(R) (1993) [nucl-th/9301006v1].

[2] C. Wilkin et al., Is there an $\eta^{3} \mathrm{He}$ quasi-bound state?, Lett. Rev. B 654, 92 (2007) [0707 .1489v1 [nucl-ex]].

[3] T. Mersmann et al., Precision study of the $\eta-{ }^{3}$ He system using the $d p \rightarrow{ }^{3}$ He $\eta$ reaction, Phys. Rev. Lett. 98, 242301 (2007) [nucl-ex/0701072v1].

[4] J. Smyrski et al., Measurement of the $d p \rightarrow{ }^{3}$ Heף reaction near threshold, Phys. Lett. B 649, 258 (2007) [nucl-ex/0702043v1].

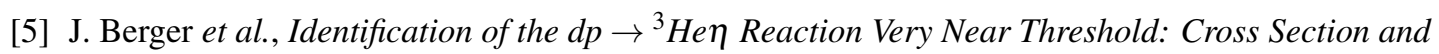
Deuteron Tensor Analyzing Power, Phys. Rev. Lett. 61, 919 (1988).

[6] B. Mayer et al., Reactions $p d \rightarrow{ }^{3} \mathrm{He \eta}$ and $p d \rightarrow{ }^{3} \mathrm{He} \pi^{+} \pi^{-}$near the $\eta$ threshold, Phys. Rev. C 53, 2068 (1996).

[7] H.-H. Adam et al., Hadronic ${ }^{3}$ Heך production near threshold, Phys. Rev. C 75, 014004 (2007).

[8] T. Rausmann et al., Precision study of the $d p \rightarrow{ }^{3}$ He $\eta$ reaction for excess energies between 20 and 60 MeV, Phys. Rev. C 80, 017001 (2009) [0905.4595v1 [nucl-ex]].

[9] R. Bilger et al., Measurement of the $\overrightarrow{p d} \rightarrow{ }^{3} \mathrm{He \eta}$ cross section between 930 and $1100 \mathrm{MeV}$, Phys. Rev. C 65, 044608 (2002).

[10] R. Bilger et al., Measurement of the pd $\rightarrow p d \eta$ cross section in complete kinematics, Phys. Rev. C 69, 014003 (2004).

[11] M. Betigeri et al., Measurement of $p+d \rightarrow{ }^{3} \mathrm{He}+\eta$ in $S_{11}$ Resonance, Phys. Lett. B 472, 267 (2000) [nucl-ex/9912006].

[12] P. Adlarson et al., Cross section ratio and angular distributions of the reaction $p+d \rightarrow{ }^{3} \mathrm{He}+\eta$ at $48.8 \mathrm{MeV}$ and 59.8 MeV excess energy, Eur. Phys. J. A 50:, 100 (2014) [1402.3469v2 [nucl-ex]].

[13] A.M. Green et al., $\eta$-Deuteron Scattering, Phys. Rev. C 54, 1970 (1996) [nucl-th/9604038v1].

[14] C. Fritzsch, "Investigation of different normalization reactions for dp collisions at the ANKE experiment", Master thesis, (2014).

[15] M. Mielke et al., Isospin effects in the exclusive $d p \rightarrow{ }^{3} H e \pi+\pi-$ reaction, Eur. Phys. J. A 50, 102 (2014) [1404.2066v1 [nucl-ex]]. 